\title{
The Entropy Production of a Nonequilibrium Open System
}

\section{Ming Bao Yu*}

407 Oak Tree Square, Athens, Georgia 30606, USA

\begin{abstract}
A nonequilibrium open system is studied in the projection operator formalism. The environment may linearly deviate from its initial state under the reaction from the open system. If the relevant statistical operator of the system is a generalized canonical one, the transport equation, the second kind of fluctuation-dissipation theorem and the entropy production rate of the open system can be derived and expressed in terms of correlation functions of fluctuations of random forces and interaction random forces.
\end{abstract}

Keywords: Non equilibrium open system; Projection operator; Entropy production rate

\section{Introduction}

In the study of nonequilibrium systems different projection operators are introduced to present a macroscopic description of the system in order to simplify the problem [1-6]. In this approach the macroscopic state of the system is determined by expectation values of a set of basis macro variables, and equations of motions for these expectation values, the transport equations, are derived in the projection operator formalism.

When studying a nonequilibrium open system, the influence of the environment upon the open system is one of the important topics in such studies. It has been shown [7] that the influence from the environment comes from two parts: one is the time-rate of the averaged macro variables resulting from the interaction Hamiltonian $H_{S R}$ and the other from an additional influence term, therefore, the influence of the environment can be completely separated from the corresponding closed system.

When the relevant statistical operator of the system is of a generalized canonical statistical operator (GCSO) by which the entropy of the open system is defined, if the environment is a reservoir, then the memory and influence terms in the transport equation can be given in terms of correlation functions of fluctuations of random forces and interacting random forces, and they can be cast into the Volterra equation formalism.

The purpose of the present paper is to generalize the results to the case that the environment is not a reservoir which may linearly deviate from its initial state under the reaction from the open system. We will show that the memory and influence terms can still be expressed in terms of correlation functions of fluctuations of random forces and interaction random forces, but no longer be able to cast into the Volterra equation formalism, so is the entropy production rate of the open system.

The results obtained in this paper are compared with approaches in linear thermodynamics and statistical mechanics, focusing on the entropy production of a nonequilibrium open system, which is local in both space and time. In contract, the entropy generation [8] is also important in the study of nonequilibrium systems, which is global in space and time, being especially useful in cases involving effects of irreversibility. In addition, another important development in physics today is the so-called quantum thermodynamics [9-14] which has extended the thermodynamics study from the macroscopic scale to the nanometer scale, and even down to the single atom and single photon scale. In Section 2, transport equations of the system are briefly reviewed. In Section 3, a GCSO is introduced. The entropy production rate is derived in Section 4. The influence term and its contribution to the entropy production is studied in Section 5. Comparison of the results with well-known approaches is presented in Section 6 and conclusions are drawn in Section 7.

\section{Transport equations}

Consider an open system $\mathrm{S}$ under the influence of its environment $\mathrm{R}$. The total system $\mathrm{S} \oplus \mathrm{R}$ is characterized by Hamiltonian $H=H_{S}+H_{R}$ $+\lambda H_{S R}$ and statistical operator (so) $W(t)$. The open system $s$ is described by a reduced statistical operator $\rho(t)=\operatorname{tr}_{R} W(t)$ satisfying $\partial \rho(t) / \partial t=-i L_{S}$ $\rho(t)+\eta(t)$ with

$$
\eta(t)=-i \lambda \operatorname{tr}_{R}\left[L_{S R} W(t)\right]
$$

describing the influence of R upon S, where $L_{S} X=i[H S, X], h=1$.

Suppose we are satisfied with the description of system $S$ at the macroscopic level by expectation values (EVs) of a set of basis macrovariables $\left\{A_{j}, j=1, \ldots, m\right\}$ of $\mathrm{S}$, such macroscopic description can be realized by a relevant so $\rho_{r}(t)$ which is picked up by a time-dependent projection operator $\rho(t)$ from $\rho(t): \rho_{r}(t)=\rho(t) \rho(t)$. We may choose the following projection operator as $\rho(t)$ [3]:

$$
p(t) X=\left[\rho_{r}(t)-\sum_{k} \frac{\partial \rho_{r}(t)}{\partial<A_{k}(t)>}<A_{k}(t)>\right] t r_{s} X+\sum_{k} \frac{\partial \rho_{r}(t)}{\left.\partial<A_{k}(t)\right\rangle} t r_{s}\left(A_{k} X\right)
$$

Introduce $q(t)=1-p(t), p(t) q(t)=0$, we have [6]

$$
\begin{aligned}
& \rho(t)=\rho_{r}(t)+g(t, 0) q(0) \rho(0)- \\
& \int_{0}^{t} d u g(t, u) q(u) i L_{s} \rho_{r}(u)+\int_{0}^{t} d u g(t, u) q(u) \eta(u)
\end{aligned}
$$

with $\dot{P}(t) \rho(t)=0 ; g(t, u)=T_{+} \exp \left\{-i \int_{u}^{t} d u_{1} q\left(u_{1}\right) L_{S}\right\}(t>u)$ is a time-ordered evolution operator satisfying

$$
\partial g(t, u) / \partial u=i g(t, u) q(u) L_{s} \text { and } g(t, t)=1
$$

*Corresponding author: Ming Bao Yu, 407 Oak Tree Square, Athens, Georgia 30606, USA, Tel: 706-410-2137; E-mail: mingbyu@gmail.com

Received April 15, 2014; Accepted January 05, 2015; Published January 10, 2015

Citation: Yu MB (2015) The Entropy Production of a Nonequilibrium Open System. J Phys Math 6: 128. doi: 10.4172/2090-0902.1000128

Copyright: (c) 2015 Yu MB. This is an open-access article distributed under the terms of the Creative Commons Attribution License, which permits unrestricted use, distribution, and reproduction in any medium, provided the original author and source are credited. 
The transport equation for $\mathrm{EV}<A_{j}(t)>=\operatorname{tr}_{s}\left[\rho(t) A_{j}\right]=\operatorname{tr}_{s}\left[\rho(t) A_{j}\right.$ takes the form [6]

$$
\frac{\partial}{\partial t}<A_{j}(t)>=\frac{\partial}{\partial t}<A_{j}(t)>^{(0)}+Y_{j}(t) \quad(\mathrm{j}=1,2, \ldots, \mathrm{m}) .
$$

In Heisenberg picture,

$$
\frac{\partial}{\partial t}<A_{j}(t)>^{(0)}=t r_{s}\left[\rho_{r}(\mathrm{t}) \dot{A}_{j}\right]+t t_{s}\left[\rho(0) Q(0) G(0, t) \dot{A}_{j}\right]+\int_{0}^{t} \operatorname{dutr}_{s}\left[\rho_{r}(u) i L_{s} Q(u) G(u, t) \dot{A}_{j}\right],
$$

here the first term gives the organized motion, the second term the initial condition and the third term the disorganized motion or the memory term [4] and

$$
Y_{j}(t)=\int_{0}^{t} d u t r_{S}\left[\eta(u) Q(u) G(u, t) \dot{A}_{j}\right]
$$

is an additional term describing the external influence from the environment upon the open system; $G(u, t) T_{-} \exp \left[i \int d u_{1} L Q\left(u_{1}\right)\right]$ $(\mathrm{t}>\mathrm{u})$ is an anti-time-ordered evolution operator defined by $\partial G(u, t) / \partial u=-i L_{S} Q(u) G(u, t)$ and $\mathrm{G}(\mathrm{t}, \mathrm{t})=1 ; Q(t)=1-P(t), P(t)$ is the transposed projection operator of $\mathrm{P}(\mathrm{t})$ [4],

$$
P(t) X=t r_{S}\left[\rho_{r}(t) X\right]+\sum_{k}\left[A_{k}-<A_{k}(t)>\right] t r_{S}\left[\frac{\partial \rho_{r}(t)}{\partial<A_{k}(t)>} X\right] ;
$$

Satisfying

$$
\operatorname{tr}_{S}[X(t) g(t, u) q(u) Y(u)]=\operatorname{tr}_{S}[Y(u) Q(u) G(u, t) X(t)]
$$

Since $\dot{A}_{j}=i\left(L_{S}+\lambda L_{S R}\right) A_{j},(2.4)$ may be written as [7]

$\frac{\partial}{\partial t}<A_{j}(t)>_{(0)}=t_{r}\left[\rho_{r}(t)\left(i L_{S} A_{j}\right)\right]+t t_{s}\left[\rho(0) Q(0) G(0, t)\left(i L_{S} A_{j}\right)\right]+\int_{0}^{t} d u t t_{s}\left[\rho_{r}(u) i L_{S} Q(u) G(u, t)\left(i L_{S} A_{j}\right)\right]$ $(\mathrm{j}=1,2, \ldots \mathrm{m})$,

Where

$\frac{\partial}{\partial t}<A_{j}(t)>_{(0)}=t_{r}\left[\rho_{r}(t)\left(i L_{S} A_{j}\right)\right]+t r_{S}\left[\rho(0) Q(0) G(0, t)\left(i L_{S} A_{j}\right)\right]+\int_{0}^{t} d u t t_{s}\left[\rho_{r}(u) i L_{S} Q(u) G(u, t)\left(i_{S} A_{j}\right)\right]$ (2.10a) is the transport equation of the corresponding closed system, i.e. the time rate of EV resulting from $H_{S}$, the Hamiltonian of the system $S$ itself; and

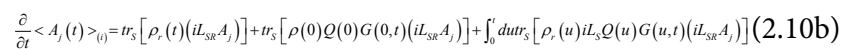

is the time rate resulting from the interaction $H_{S R}$.

The meaning of (2.9) is clear and simple: The transport equation of an open system is the sum of transport equation of the corresponding closed system, the time-rate of the EV due to the interaction Hamiltonian $H_{S R}$ and the additional influence term $Y_{j}(t)$.

The influence term (2.6) can be written as $(\mathrm{j}=1,2 \ldots, \mathrm{m})(\mathrm{j}=1,2 \ldots, \mathrm{m}), \quad(2.11)$

Where $f_{j}(u, t)=Q(u) G(u, t) \dot{A}_{j}$ denotes the random force, which may be split into two:

$$
\begin{aligned}
& f_{j}(u, t)=f_{j}^{s}(u, t)+\lambda f_{j}^{S R}(u, t), \\
& f_{j}^{S}(u, t)=Q(u) G(u, t)\left(i L_{S} A_{j}\right), \\
& f_{j}^{S R}(u, t)=Q(u) G(u, t)\left(i L_{S R} A_{j}\right)
\end{aligned}
$$

being respectively the random force and interaction random force associated with the time rate of the basis variable $A_{j}$ due to $H_{S}$ and $H_{S R}$, respectively. Since the average of the random force over given ensembles vanishes, so $f_{j}(u, t)=\delta f_{j}(u, t)$ In the rest of the paper we will no longer distinguish $f_{j}(u, t)$ from its fluctuation $\delta f_{j}(u, t)$.

\section{Generalized canonical statistical operator In order to go steps further}

let us assume $\rho_{r}(t)$ to be a GCSO:

$$
\rho_{r}(t)=e^{-\sum_{l} \lambda_{l}(t) A_{l}} / z_{r}(t)=\operatorname{tr}_{S}\left[e^{-\sum_{l} \lambda_{l}(t) A_{l}}\right]
$$

where $\lambda_{t}$ (t) $(l=1, \ldots, m)$ are conjugate parameters of the basis macrovariables $\left\{A_{t}\right\}$

Making use of the Kubo identity $\left[X, e^{Y}\right]_{-}=\int_{0}^{1} d \alpha e^{\alpha Y}[X, Y]_{-} \mathrm{e}^{(1-\alpha) Y}$ we have

$$
\begin{aligned}
& i L_{S} \rho_{r}(t)=-\sum_{l} \int_{0}^{1} d \alpha e^{-\alpha \sum_{l} \lambda_{l}(t) A_{l}}\left(i L_{S} A_{l}\right) e^{\alpha \sum_{l} \lambda_{l}(t) A_{l}} \rho_{r}(t) \lambda_{l}(t) \\
& i L_{S R} \rho_{r}(t)=-\sum_{l} \int_{0}^{1} d \alpha e^{-\alpha \sum_{l} \lambda_{l}(t) A_{l}}\left(i L_{S R} A_{l}\right) e^{\alpha \sum_{l} \lambda_{l}(t) A_{l}} \rho_{r}(t) \lambda_{l}(t)
\end{aligned}
$$

Introducing the generalized quantum correlation function

$$
(X(t), Y(u))_{r}=\int_{0}^{1} d \alpha t r_{S}\left[X(t) e^{-\alpha \sum_{l} \lambda_{l}(u) A_{l}} Y(u) e^{\alpha \sum_{l} \lambda_{l}(u) A_{l}} \rho_{r}(u)\right]
$$

and making use of (3.2), the integrand in (2.5) may be written as

$$
\operatorname{tr}_{S}\left[\rho_{r}(u) i L_{S} f_{j}(u, t)\right]=\sum_{l}\left(f_{j}(u, t), i L_{S} A_{l}\right)_{r} \lambda_{l}(u)
$$

Since $f_{j}(u t)$ is in the irregular space because of $Q(u)$ while is in the regular space, their correlation is zero, thus

$$
\operatorname{tr}_{S}\left[\rho_{r}(u) i L_{S} f_{j}(u, t)\right]=\sum_{l}\left(f_{j}(u, t), f_{l}^{S}(u, u)\right)_{r} \lambda_{l}(u)
$$

Where $f_{l}^{S}(u, u)$ is given by $(2.12 \mathrm{~b})$. Same argument will apply to similar cases later.

Therefore Eq.(2.5) takes the form

$$
\frac{\partial}{\partial t}<A_{j}(t)>^{(0)}=t_{s}\left[\rho_{r}(t) \dot{A}_{j}\right]+t t_{s}\left[\rho(0) Q(0) G(0, t) \dot{A}_{j}\right]+\sum_{l} \int_{0}^{t} d u\left(f_{j}(u, t), f_{l}^{s}(u, u)\right)_{r} \lambda_{l}(u)
$$

here the memory term is expressed in terms of quantum correlation function of fluctuations of random forces. The influence term $Y_{j}(t)$ will be further analysed in Section 5 .

\section{Entropy production rate}

Now define the entropy of the noequilibrium open system through its relevant statistical operator $[1,15,16]$

$$
S(t)=-k_{B} t r_{S}\left[\rho_{r}(t) \ln \rho_{r}(t)\right]
$$

where $k_{B}$ is the Boltzmann constant. The entropy production rate reads [7]

$$
\frac{\partial S(t)}{\partial t} \quad k_{B} \sum \frac{\partial<A(t)>}{\partial t}{ }_{j}(t)
$$

which is the sum of products of transport equations and the conjugate parameters. If assume that the initial state of the system is a GCSO: $\rho$ $(0)=\rho_{r}(0)$ then the initial term in (3.6) vanishes. Combining (4.2) with (2.4) given by (3.6) and (2.11), we obtain

$$
\frac{\partial S_{1}(t)}{\partial t}=\frac{\partial S_{1}(t)}{\partial t}+\frac{\partial S_{2}(t)}{\partial t}+\frac{\partial S_{3}(t)}{\partial t},
$$

the first term resulting from the organized motion in (3.6) reads [7] 


$$
\frac{\partial S_{1}(t)}{\partial t}=\lambda k_{B} \sum_{j=1}^{m} \lambda_{j}(t) t r_{S}\left[\rho_{r}(t) t r_{S}\left(i L_{S R} A_{j}\right)\right]
$$

the second term resulting from the disorganized motion in (3.6) takes the form

$$
\frac{\partial S_{2}(t)}{\partial t}=k_{B} \sum_{j, 1} \int_{0}^{t} d u\left(f_{j}(u, t), f_{l}^{S}(u, u)\right)_{r} \lambda_{l}(u) \lambda_{j}(t)
$$

because of (3.5); and the third term resulting from the influence term (2.11) is

$$
\frac{\partial S_{3}(t)}{\partial t}=k_{B} \sum_{j} \int_{0}^{t} d u t r_{S}\left[\eta(u) f_{j}(u, t)\right] \lambda_{j}(t)
$$

These expressions represent the contributions of each term in the transport equation to the entropy production, respectively. Besides, Eq.(4.4) does not involve $i L_{S} A$, indicating that in the organized motion term $H_{S}$ contributes nothing to the rate.

\section{Non-reservoir environment}

Now we further analyze the contribution of the influence term $Y_{j}(t)$. Suppose that the environment $\mathrm{R}$ is not a reservoir and may linearly deviate from its initial state under the reaction from $\mathrm{S}$. For simplicity, we assume $H_{S R}=\sum_{k} \gamma_{k} A_{k} B_{k}, A_{k}$ and $B_{k}$ respectively pertain to $\mathrm{S}$ and R, and they are initially independent:

$$
W(0)=\rho(0) R(0) \text { where } R(t)=t_{S} W(t) .
$$

By $W(0)=\rho(0) R(0)$, we have $W(u)=\rho(u) R(u)$, here $\rho(u)=e^{-i\left(L_{S}+\lambda L_{S R}\right) u} \rho(0)$ and $R(u)=e^{-i\left(L_{S}+\lambda L_{S R}\right) u} R(0)$. By (2.1), $\eta(u)$ may be written as

$$
\begin{aligned}
& \eta(u)=-i \lambda<L_{S R}(u)>_{R 0} \rho(u)=-i \lambda\left[<H_{S R}(u)>_{R 0}, \rho_{r}(t)\right]_{-} \\
& <H_{S R}(u)>_{R 0}=\sum_{k} \gamma_{k} A_{k}<B_{k}(u)>_{R 0,} \\
& <B_{k}(u)>_{R 0}=\operatorname{tr}_{R}\left[B_{k}(u) R(0)\right]
\end{aligned}
$$$$
\left.<B_{k}(u)\right\rangle_{R 0} \text { determines the evolution of the EV of macrovariable }
$$
$B_{k}$

Since $\partial B_{k}(t) / \partial t=i L_{R} B_{k}(t)+i \lambda L_{S R} B_{k}(t)$ thus $B_{k}(t)=e^{i L_{R} t} B_{k}(0)+i \lambda \int_{0}^{t} d u e^{i L_{R}(t, u)} L_{S R} B_{k}(u)$

For weak interaction, keeping only the linear term in $\lambda$, we obtain

$$
\begin{aligned}
& B_{k}(t)=e^{i L_{R} t}\left[1+i \lambda \int_{0}^{t} d u L_{S R}(u)\right] B_{k}(0)+o\left(\lambda^{2}\right), L_{S R}(u)=e^{i L_{R} u} L_{S R} e^{i L_{R} u} \\
& <B_{k}(u)>_{R 0}=<B_{k}(u)>_{R 0}^{(0)}+B_{k}(u)>_{R 0}^{(1)} \\
& <B_{k}(u)>_{R}^{()}=\operatorname{tr}_{R}\left[R(0) e^{i L u} B_{k}(0)\right] \\
& <B_{k}(u)>_{R 0}^{(1)}=i \lambda \operatorname{tr}_{R}\left[R(0) e^{i L_{R} u} \int_{0}^{u} d u_{1} L_{S R}\left(u_{1}\right) B_{k}(0)\right]
\end{aligned}
$$

being the zeroth and first order terms of the $\mathrm{EV}$ of $B_{K}$ when R linearly deviates from its initial state under the weak reaction from S. By (5.1) and (5.2), the integrand in (2.11) takes the form

$$
\operatorname{tr}_{S}\left\{\eta(u) f_{j}(u, t)\right\}=-i \lambda \sum_{k} \gamma_{k} t r_{S}\left\{f_{j}(u, t)\left[A_{k}, \rho(u)\right]_{-}\right\}<B_{k}(u)>_{R 0}
$$

$$
=-i \lambda \sum_{k} \gamma_{k} t r_{S}\left\{F_{j}(u, t)\left[A_{k}(u), \rho(0)\right]_{-}\right\}<B_{k}(u)>_{R 0}
$$

in Schrodinger and Heisenberg pictures, respectively; where

$F_{j}(u, t)=e^{i\left(L_{S}+L_{S R}\right) u} f_{j}(u, t)$ and $A_{k}(u)=e^{i\left(L_{S}+L_{S R}\right) u} A_{k}$. Hence we have the influence term

$$
\begin{aligned}
& \left.Y_{j}(t)=-i \lambda \sum_{k} \gamma_{k} \int_{0}^{t} d u t r_{S}\left\{f_{j}(u, t)\left[A_{k}, \rho(u)\right]_{-}\right\}<B_{k}(u)\right\rangle_{R 0} \\
& \left.=-i \lambda \sum_{k} \gamma_{k} \int_{0}^{t} d u t r_{S}\left\{F_{j}(u, t)\left[A_{k}(u), \rho(0)\right]_{-}\right\}<B_{k}(u)\right\rangle_{R 0}
\end{aligned}
$$

and its contribution to the entropy production in Shrodinger and Heisenberg pictures:

$$
\begin{aligned}
& \left.\frac{\partial S_{3}(t)}{\partial t}=-i \lambda k_{B} \sum_{j, k} \gamma_{k} \int_{0}^{t} d u t r_{S}\left\{f_{j}(u, t)\left[A_{k}, \rho(u)\right]_{-}\right\}<B_{k}(u)\right\rangle_{R 0} \lambda_{j}(t) \\
& \left.-i \lambda k_{B} \sum_{j k} \gamma_{k} \int d u t r_{S}\left\{F_{j}(u, t)\left[A_{k}(u), \rho(0)\right]_{-}\right\}<B_{k}(u)\right\rangle_{R} \lambda_{j}(t)
\end{aligned}
$$

Now consider the case that the initial state of $\mathrm{S}$ is given by a GCSO:

$$
\rho_{r}(0)=e^{-\sum_{l} \lambda_{l}(0) A_{l}} / t r_{S} e^{-\sum_{l} \lambda_{l}(0) A_{l}} .
$$

By the Kubo identity and the initial condition $\rho(0)=\rho_{r}(0)$ we have

$$
\left[A_{k}(u), \rho(0)\right]_{-}=-\sum_{l} \lambda_{l}(0) \int_{0}^{1} d \alpha e^{-\alpha \sum_{l} \lambda_{l}(0) A_{l}} \rho_{r}(0) \text {, }
$$

Thus (5.4) may be written as

$\operatorname{tr}_{S}\left\{\eta(u) f_{j}(u, t)\right\}=\lambda \sum_{l}\left(F_{j}(u, t), i<L_{S R}(u)>_{R 0} A_{l}\right)_{r 0} \lambda_{l}(0)=\lambda \sum_{l}\left(F_{j}(u, t), f_{l}^{-S R}(u, u)\right)_{r 0} \lambda_{l}(0)$ here we have conducted argument similar to that leads (3.4) to (3.5), and

$$
\stackrel{-S R}{f_{l}}(u, u)=Q(u) G(u, u)\left[i<L_{S R}(u)>_{R 0} A_{l}\right]
$$

is the averaged interaction random force. Thus we obtain

$$
\begin{aligned}
& Y_{j}(t)=\lambda \sum_{l} \int_{0}^{t} d u\left(F_{j}(u, t), f_{l}^{-S R}(u, u)\right)_{r 0} \lambda_{l}(0) \\
& \frac{\partial S_{3}(t)}{\partial t}=\lambda k_{B} \sum_{j, l} \int_{0}^{t} d u\left(F_{j}(u, t), f_{l}^{-S R}(u, u)\right)_{r 0} \lambda_{l}(0) \lambda_{j}(t) \\
& \text { With }(X(t), Y(u))_{r 0}=\int_{0}^{1} d \alpha t r_{S}\left\{X(t) e^{-\alpha \sum_{l} \lambda_{l}(0) A_{l}} \rho_{r}(0)\right\}
\end{aligned}
$$

If the open system is initially in an equilibrium state

$$
\rho(0)=\rho_{r}(0)=\rho_{e q}=e^{-\beta_{0} H_{S}} / \operatorname{tr}_{S}\left(e^{-\beta_{0} H_{S}}\right),
$$

$\beta_{0}=1 / k_{B} T_{0}$ is the initial inverse temperature of the system, then $\rho_{e q}$ is a special case of (5.7) in which $A_{1}=H_{S}, \lambda_{1}(0)=\beta_{0}$ and $A_{1}=0(l \geq 2)$. Since $A_{1}$ is the only basic variable, so $\rho_{r}(t)=e^{-\beta(t) H_{S}} / t r_{S}\left[e^{-\beta(t) H_{S}}\right]$ and $\beta(t)=1 / k_{B} T(t)$ is the inverse temperature of S. Because $i L_{S} H_{S}=0$, thus $\dot{H}_{S}=i \lambda L_{S R} H_{S}$ and $f_{1}^{S}(u, t)=0, f_{1}(u, t)=\lambda f_{1}^{S R}(u, t)$;

the memory term in (3.6) becomes $\lambda \int_{0}^{t} d u\left(f_{1}^{S R}(u, t), f_{1}^{S}(u, u)\right)_{r} \beta(u)$ and vanishes, so 


$$
\frac{\partial<H_{S}(t)>^{(0)}}{\partial t}=\lambda t r_{S}\left[\rho_{r}(t)\left(i L_{S R} H_{S}\right)\right]
$$

Besides, as a special case of (5.9) and (5.10), we have

$$
\begin{aligned}
& Y_{1}(t)=\lambda^{2} \beta_{0} \int_{0}^{t} d u\left(F_{1}^{S R}(u, t), f_{1}^{-S R}(u, u)\right)_{e q} \\
& \frac{\partial S_{3}(t)}{\partial t}=\frac{\lambda^{2}}{T_{0}} \int_{0}^{t} d u\left(F_{1}^{S R}(u, t), f_{1}^{-\mathrm{SR}}(u, u)\right)_{e q} \beta(t)
\end{aligned}
$$

Here $(X(t), Y(u))_{e q}=\int_{0}^{1} d \alpha t r_{S}\left\{X(t) e^{-\alpha \beta_{0} H_{S}} Y(u) e^{-\alpha \beta_{0} H_{S}} \rho_{e q}\right\}$

Finally we obtain the transport equation for the only basis variable $H_{S}$ :

$$
\frac{\partial S(t)}{\partial t}=\frac{\lambda}{T(t)} \operatorname{tr}_{S}\left[\rho_{r}(t)\left(i L_{S R} H_{S}\right)\right]+\frac{\lambda^{2}}{T_{0}} \int_{0}^{t} d u\left(F_{1}^{S R}(u, t), f_{1} \overline{(}(u, u)\right)_{e q} \beta(t)
$$

Eqs.(5.9), (5.10) and (5.14), (5.15) involve the averaged interaction random force (5.8) which has incorporated the linear deviation of the environment from its initial state.

Now consider the case without a given initial condition. By (3.1) and the Kubo identity, we have

$$
i<L_{S R}(u)>_{R 0} \rho_{r}(u)=-\sum_{l} \lambda_{l}(u) \int_{0}^{1} d \alpha e^{-\alpha \sum_{l}^{\lambda_{r}(u) A_{4}}}\left[i<L_{S R}(u)>_{R 0} A_{l}\right] e^{-\alpha \sum_{l} \lambda_{r}(u) A_{4}} \rho_{r}(u)
$$

With (5.1) and (2.3),

$$
\operatorname{tr}_{S}\left\{\eta(u) f_{j}(u, t)\right\}=-\lambda t r_{S}\left[f_{j}(u, t) i<L_{S R}(u)>_{R 0} \rho(u)\right]=J_{1}+J_{2}+J_{3}
$$

Eq.(5.19) is similar to Eq.(22) in [17] where the environment is a reservoir and $\left\langle L_{S R}\right\rangle_{R 0}$ is time-independent. In the following, we will follow the argument in [17], however, take into consideration that $<L_{S R}(u)>_{R 0}$ is time-dependent. Making use of (5.18) and (3.3), leads to

$$
\begin{aligned}
& J_{1}=-\lambda t r_{S}\left\{f_{j}(u, t) i<L_{S R}(u)>_{R 0} \rho_{r}(u)\right\}=\lambda \sum_{l} \lambda_{l}(u)\left(f_{j}(u, t), i<L_{S R}(u)>_{R 0} A_{l}\right)_{r} \\
& J_{2}=\lambda \int_{0}^{u} d u_{1} t r_{S}\left\{f_{j}(u, t) i<L_{S R}(u)>_{R 0} \tilde{K}_{1}\left(u, u_{1}\right) \rho_{r}\left(u_{1}\right)\right\} \\
& =\lambda \sum_{l} \int_{0}^{u} d u_{1} \lambda_{1}\left(u_{1}\right)\left(K_{1}\left(u_{1}, u\right) f_{j}(u, t), i<L_{S R}(u)>_{R 0} A_{l}\right)_{r} \\
& \tilde{K}_{1}\left(u, u_{1}\right)=g\left(u, u_{1}\right) q\left(u_{1}\right) i L_{S} \\
& K_{1}\left(u_{1}, u\right)=i L_{S} Q\left(u_{1}\right) G\left(u_{1}, u\right) \\
& J_{3}=-\lambda \int_{0}^{u} d u_{1} t r_{S}\left[f_{j}(u, t) i<L_{S R}(u)>_{R 0} g\left(u, u_{1}\right) q\left(u_{1}\right) \eta\left(u_{1}\right)\right] \\
& =\lambda^{2} \int_{0}^{u} d u_{1} t r_{S}\left\{\rho\left(u_{1}\right) \tilde{K}\left(u_{1}, u\right) \tilde{K}(u, t) \dot{A}_{j}\right\} \\
& \tilde{K}(u, t)=i<L_{S R}(u)>_{R 0} Q(u) G(u, t) .
\end{aligned}
$$

Substituting (2.3) into (5.23) and repeating the above arguments, we have

$$
\begin{aligned}
& \operatorname{tr}_{S}\left[\eta(u) f_{j}(u, t)\right]=\lambda \sum_{l}\left\{\lambda_{l}(u)\left(f_{j}(u, t), i<L_{S R}(u)>_{R 0} A_{l}\right)_{r}+\right. \\
& \left.\int_{0}^{u} d u_{1} \lambda_{1}\left(u_{1}\right)\left(K_{1}\left(u_{1}, u\right) f_{j}(u, t), i<L_{S R}(u)>_{R 0} A_{l}\right)_{r l}+\ldots\right\}
\end{aligned}
$$

$$
\begin{aligned}
& +\lambda^{2} \sum_{l} \int_{0}^{u} d u_{1} \lambda_{l}\left(u_{1}\right)\left(K\left(u_{1}, u\right) f_{j}(u, t), i<L_{S R}(u)>_{R 0} A_{l}\right)_{r} \\
& \left.+\int_{0}^{u_{1}} d u_{2} \lambda_{1}\left(u_{2}\right)\left(K_{1}\left(u_{2}, u_{1}\right) f_{j}(u, t), i<L_{S R}\left(u_{1}\right)>_{R 0} A_{l}\right)_{r l}+\ldots\right\} \\
& K\left(u_{1}, u\right)=Q\left(u_{1}\right) G\left(u_{1}, u\right) i<L_{S R}(u)>_{R 0},
\end{aligned}
$$

which many be written as

$$
\begin{aligned}
& \operatorname{tr}_{S}\left[\eta(u) f_{j}(u, t)\right]=\lambda \sum_{l}\left\{\lambda_{1}(u)\left(f_{j}(u, t), f_{1}^{-S R}(u, u)\right)_{r}+M_{1}(u, t)\right\} \quad(5.26) \\
& M_{1}(u, t)=\sum_{n=1}^{\infty} \lambda^{n-1} \int_{0}^{u} d u_{1} \ldots \int_{0}^{u_{n-1}} d u_{n} \lambda_{1}\left(u_{n}\right)\left[\left(K_{1}\left(u_{n-1}, u_{n-2}\right) \ldots K\left(u_{1}, u\right) f_{j}(u, t), f_{1}^{-S R}\left(u_{n-1}, u_{n-1}\right)\right)_{r}\right. \\
& \left.+\lambda K\left(u_{n}, u_{n-1}\right) K\left(u_{n-1}, u_{n-2}\right) \ldots K\left(u_{1}, u\right) f_{j}(u, t), f_{l}^{-S R}\left(u_{n}, u_{n}\right)_{r}\right]
\end{aligned}
$$

here we have had argument similar to that leads (3.4) to (3.5). Thus we obtain the influence term and its contribution to the entropy production:

$$
\begin{aligned}
& Y_{j}(t)=\lambda \sum_{l} \int_{0}^{t} d u\left\{\lambda_{1}(u)\left(f_{j}(u, t), f_{l}^{-\mathrm{SR}}(u, u)\right)_{r}+M_{1}(u, t)\right\} \\
& \frac{\partial S_{3}(t)}{\partial t}=\lambda k_{B} \sum_{j, l} \int_{0}^{t} d u\left\{\lambda_{l}(u)\left(f_{j}(u, t), f_{l}^{-S R}(u, u)\right)_{r}+M_{l}(u, t)\right\} \lambda_{j}(t)
\end{aligned}
$$

If we are satisfied with keeping the linear term of $\lambda$ in $M_{l}(u, t)$, then

$$
\begin{aligned}
& M_{l}(u, t)=\int_{0}^{u} d u_{1} \lambda_{l}\left(u_{l}\right)\left(K_{1}\left(u_{1}, u\right) f_{j}(u, t), f_{l}(u, u)\right)_{r}^{S R}+o\left(\lambda^{2}\right) \\
& \operatorname{tr}_{S}\left[\eta(u) f_{j}(u, t)\right]=\lambda \sum_{l}\left(\phi(u) f_{j}(u, t), f_{l}^{-S R}(u, u)\right)_{r}+o\left(\lambda^{3}\right) \\
& \phi_{1}(u)=\lambda_{l}(u)+\int_{0}^{u} d u_{1} \lambda_{l}\left(u_{1}\right) K_{1}\left(u_{1}, u\right)
\end{aligned}
$$

Therefore we have the approximate expressions

$$
\begin{aligned}
& Y_{j}(t)=\lambda \sum_{l} \int_{0}^{t} d u\left(\phi_{l}(u) f_{j}(u, t), f_{l}^{-S R}(u, u)\right)_{r} \\
& \frac{\partial S_{3}(t)}{\partial t}=\lambda k_{B} \sum_{j, l} \int_{0}^{t} d u\left(\phi_{l}(u) f_{j}(u, t),{ }^{-S R} f_{l}(u, u)\right)_{r} \lambda_{j}(t)
\end{aligned}
$$

they are up to $\lambda^{2}$ by (2.12a). Comparing (5.34) with (4.5), we see clearly that $\phi_{l}(u)$ plays the role of $\lambda_{l}(u)$ in the case of corresponding isolate system.

Now we rewrite the results obtained above in the form of special dependent. For simplicity, we focus on the simpler expression (5.34). The entropy production of the open system reads

$$
\frac{\partial s(x, t)}{\partial t}=\frac{\partial s_{1}(x, t)}{\partial t}+\frac{\partial s_{2}(x, t)}{\partial t}+\frac{\partial s_{3}(x, t)}{\partial t}
$$

Where

$$
\frac{\partial s_{1}(x, t)}{\partial t}=\lambda k_{B} \sum_{j=1}^{m} \lambda_{j}(x, t) t r_{S}\left[\rho_{r}(x, t)\left(i L_{S R} A_{j}\right)\right]
$$

results from the organized motion in the transport equation due to $H_{S R} ;$ 


$$
\frac{\partial s_{2}(x, t)}{\partial t}=k_{B} \sum_{j, l} \int_{0}^{t} d u\left(f_{j}(u, t), f_{l}^{S}(u, u)\right)_{r} \lambda_{l}(x, u) \lambda_{j}(x, t)(5.36 \mathrm{~b})
$$

from the disorganized motion and

$$
\frac{\partial s_{3}(x, t)}{\partial t}=\lambda k_{B} \sum_{j, l} \int_{0}^{t} d u\left(\phi_{l}(x, u), f_{j}(u, \mathrm{t})\right), f_{l}^{-S R}(\mathrm{u}, u)_{r} \lambda_{j}(x, t)
$$

from the influence term, respectively.

\section{Comparison}

In this section, we compare the results obtained in the proceeding sections with the well known approaches in the linear nonequilibrium thermodynamics and statistical mechanics. The time rate of the entropy density $s(x, t)$ of a nonequilibrium system takes the form $[8,18,19]$ :

$$
\begin{aligned}
& \frac{\partial s(x, t)}{\partial t}=\sigma(x, t)-\nabla \cdot j_{s}(x, t) \\
& \text { Where } \sigma(x, t)=\sum_{i} X_{i}(x, t) J_{i}(x, t)
\end{aligned}
$$

is the entropy production density occurring inside the system which is given in terms of the sum of products of thermodynamic fluxes $J_{i}(x, t)$ and the conjugate thermodynamic forces $X_{i}(x, t)$; and $j_{s}(x, t)$ is the density of entropy flux through the border into the system. forces

Onsager proposed a linear relationship between the fluxes and

$$
J_{i}(x, t)=\sum_{k} L_{i k} X_{k}(x, t)
$$

with reciprocity relations

$$
L_{i k}=L_{k i}
$$

Thus we have

$$
\frac{\partial s(x, t)}{\partial t}=\sum_{i, k} L_{i k} X_{i}(x, \mathrm{t}) X_{k}(x, t)-\nabla \cdot \mathrm{j}_{s}(x, t)
$$

For the special case considered in Sect.5, the interaction between open system $\mathrm{S}$ and its environment $\mathrm{R}$ takes the form $H_{S R}=\sum_{k} \gamma_{k} A_{k} B_{k}$, for example, $\mathrm{S}$ and $\mathrm{R}$ are composed of different kinds of harmonic oscillator [7]. Such interaction implies no obvious border separating $\mathrm{S}$ and $\mathrm{R}$, leading to absence of the divergence term on the right hand side of (6.5). Thus the variation of entropy density results from inner entropy production $\sigma(x, t)$ only:

$$
\frac{\partial s(x, t)}{\partial t}=\sum_{i, k} L_{i k} X(x, t)_{i} X_{k}(x, t)
$$

Besides, in the Green-Kubo formalism, the transport coefficients $L_{i k}$ can be expressed in terms of time correlation functions of the time rate of corresponding variables $[19,20]$.

$$
L_{i k} \sim \int_{0}^{\infty} d t<\dot{A}_{i}(t) \dot{A}_{k}(0)>_{e q}
$$

where the average is taken over an equilibrium ensemble and the Markovian effect is taken into account.

In this paper, we study a nonequilibrium open system whose transport equations (2.9)-(2.11) are nonlinear differential-integral ones. Now let us compare (5.36b) with (6.6). We notice that (5.36b) share the same structure as (6.6) because of the facts : (1) parameters $\left\{\lambda_{j}(x, t)\right\}(\mathrm{j}=1, \ldots \mathrm{m})$ play the role Thermodynamic forces since they may involve spacial gradients of, e.g., temperature, velocity, chemical potential or electric, magnetic fields, etc,; (2) the random forces (2.12b) involve the time rates of variables because of using projection operator technique and (3) the average is taken over GCSO (3.1) instead of an equilibrium ensemble. As for (5.36c), the contribution of $Y_{j}(t)$ to the entropy production, in which the free term $\phi_{l}(x, u)$ in the Volterra equation is indeed a generalization of $\lambda_{l}(x, u)$ in (5.26b), hence (5.36c) possesses the same structure as (6.6) also. Acoordingly, we see clearly that the entropy production rate (5.35) is a natural generalization of (6.6) where the non-linearity and the non-Markovian effect have been taken into consideration. In addition to the entropy production, the entropy generation is another useful tool in the study of nonequilibrium systems [8] and especially useful in the analysis of a process occurring in the system during a period of time $\tau$. It is worth noticing the major differences between the two: the entropy production needs the hypothesis of local equilibrium but the entropy generation does not; the former does not consider the time but the latter introduces the lifetime $\tau$ of the process [8]. The two different approaches are closely related and complementary one to another.

\section{Conclusion}

In the present paper we have studied a nonequilibrium open system in interaction with its environment which may linearly deviate from its initial state under the reaction of the open system. We have shown that if the relevant statistical operator of the system is of the form of GCSO, then the transport equation is given by (3.6) and (5.28) or (5.33). The memory term in (3.6) and the influence term (5.28) or (5.33) can be expressed in terms of quantum correlation functions of fluctuations of random forces and interaction random forces, giving the second kind of fluctuation-dissipation theorem for this nonequilibrium open system. We have also shown that the entropy production rate is given by the sum of products of transport equations and the corresponding parameters. In the organized motion term, $H_{S}$ contributes nothing to the rate, but $H_{S R}$ does; the contributions of the memory and influence terms are expressed in terms of quantum correlation functions of fluctuations of random forces and interaction random forces. The total entropy production rate is given by the sum of contributions resulting from each term in the transport equation, given respectively by (4.4), (4.5) and (5.29) or (5.34). They are natural generalizations of those for a linear nonequilibrium closed system to a nonlinear open system.

\section{References}

1. Robertson B (1996) Equations of Motion in Nonequilibrium Statistical Mechanics. Phys Rev 144: 151.

2. Li KH (1986) Physics of open systems. Phys Rep 134: 1-85.

3. Kawasaki K, Gunton JD (1973) Phys Rev A 8: 2048.

4. Grabert H (1978) Projection Operator Techniques in Nonequilibrium Statistical Mechanics.J Stat Phys 19: 479

5. Oppenheim I, Levin RD (1979) Nonlinear Transport Processes: Hydrodynamics Physica A 99: 383-402

6. Yu MB (1986) Physica A 137

7. Yu MB (2008) Influence of environment and entropy production of a nonequilibrium open system. Phys Lett A 372: 2572-2577.

8. Lucia U (2013) Stationary open systems: A brief review on contemporary theories on irreversibility. Physica A 392: 1051-1062.

9. Hatsopoulos GN, Gyftopoulos EP (1976) A Unified Quantum Theory of Mechanics and Thermodynamics. Part I. Postulates. Foundations of Phys 6: 15.

10. Hatsopoulos GN, Gyftopoulos EP (1976) A Unified Quantum Theory of 
Mechanics and Thermodynamics. Part Ila. Available Energy. Foundations of Phys 6: 127

11. Hatsopoulos GN, Gyftopoulos EP (1976) A Unified Quantum Theory of Mechanics and Thermodynamics. Part Ilb. Stable Equilibrium States. Foundations of Phys 6: 439

12. Hatsopoulos GN, Gyftopoulos EP (1976) A Unified Quantum Theory of Mechanics and Thermodynamics. Part III Irreducible Quantal Dispersions. Foundations of Phys 6: 561.

13. Beretta GP (2010) Maximum entropy production rate in quantum thermodynamics. J Phys: Conference Series 237: 1-32.

14. Sciacovelli A, Smith CE, Vonspakovsky MR, Verda V (2010) Quantum Thermodynamics: Non-equilibrium 3D Description of and Unbounded System at an Atomistic Level. Int $\mathrm{J}$ of Thermodynamics 13: 23-33.
15. Nettleton RE (2001) Positive definiteness of entropy production in the nonlinear Robertson formalism. J Chem Phys 14: 6007-6013.

16. Jarvis JB (2005) Time-dependent entropy evolution in microscopic and macroscopic electromagnetic relaxation. Phys Rev E 72 .

17. MB Yu (1990) Phys Lett A 150: 1-10.

18. Groot SD, Mazur P (1962) Non-Equilibrium Thermodynamics, North-Holland.

19. Martyushev LM, Seleznev VD (2006) The restrictions of the Maximum Entropy Production Principle. Phys Rept 426: 1-45.

20. Kubo R (1957) The Glass Transition: Relaxation Dynamics in Liquids and Disordered Materials. J Phys Soc Jpn 12: 570-586.
Citation: Yu MB (2015) The Entropy Production of a Nonequilibrium Open System. J Phys Math 6: 128. doi: 10.4172/2090-0902.1000128
Submit your next manuscript and get advantages of OMICS Group submissions

Unique features:

- User friendly/feasible website-translation of your paper to 50 world's leading languages

- Audio Version of published paper

Digital articles to share and explore

Special features:

400 Open Access Journals

30,000 editorial team

21 days rapid review proces

Quality and quick editorial, review and publication processing

Indexing at PubMed (partial), Scopus, EBSCO, Index Copernicus and Google Scholar etc

- Sharing Option: Social Networking Enabled

- Authors, Reviewers and Editors rewarded with online Scientific Credits

- Better discount for your subsequent articles

Submit your manuscript at: http://www.omicsonline.org/submission/ 